\title{
Study of the Effect of RCS on Radar Detection
}

\author{
Dr. Haitham Kareem Ali (Assistant Professor) \\ Technical College of Engineering, \\ Sulaimani Polytechnic University, Kurdistan Region, Iraq
}

doi: 10.19044/esj.2017.v13n15p148 URL:http://dx.doi.org/10.19044/esj.2017.v13n15p148

\begin{abstract}
Objectives: To study the effect of the radar cross section (RCS) area for the target on the radar operation, and to show its performance on radar detection by taking samples from the RCS. Methods/Statistical Analysis: In this paper, we tested the effect of RCS on radar detection under two features of RCS using radar lab. These features are: (i) shapes with constant area and (ii) shapes with different areas. Findings: Experimental testing of the radar delectability in order to know its capability in detecting targets under and above two features( by measuring the power of echo signal) shows that whenever the power of echo signal is high (i.e. RCS is an excellent reflector), the radar detection range is high and vice versa. Application/Improvements: This proposed approach can be used in designing the target bodies using different shapes (i.e. reflectors) to dispute the transmitted signal and to weaken it. This makes the detection of targets extremely hard.
\end{abstract}

Keywords: Signal, antenna, radar system, reflection, target

\section{Introduction}

The ability of the radar system to detect and display a given target depends on a large number of factors. Some of those factors are constant and others may vary in a quite complex manner. The factors can be classified as significant factors and non-significant factors. The radar range equation is formulated as the relation between the maximum range at which a target can be detected and the parameters that the range depends on. Based on the form in which the equation is used by radar engineers to predict detection ranges of targets by taking into account all conceivable factors, it is indeed lengthy and complex. Also, it contains statistical and empirical components. However, in its simplest form as set out below, it provides at a glance an indication of the significance of many of the factors which are of concern to the radar observer: 


$$
R \max =\sqrt[4]{\left(\frac{P t \cdot G \cdot A \cdot \sigma}{4 \pi^{2} S \min }\right)}
$$

Where:

$\mathrm{R}_{\max }=$ maximum detection range

$\mathrm{P}_{\mathrm{t}}=$ transmitter power

$\mathrm{G}=$ antenna gain

$\mathrm{A}=$ antenna aperture area $\left(\mathrm{m}^{2}\right)$

$\sigma=$ target radar cross section $\left(\mathrm{m}^{2}\right)$

$\mathrm{S}_{\min }=$ minimum detectable signal

Consequently, only one of the variables is related to the characteristics of the radar system. The sole representative of the target is its radar cross section, which measure the size of the target as 'seen' by the radar. Except in the case of certain simple shapes, this is a complex quantity, often having a statistical nature. The simple form of the radar equation assumes that the radar and the target are in free space. In practice, they are both located on a curved earth having a surface of varying character and surrounded by an atmosphere in which various weather effects may be manifested. The simple equation takes into account (i) the surface over which, and (ii) the medium through which, the radar pulse travels. This is because many of the effects are extremely difficult to quantify (Merrill Skolnik, 2002; Fred et al., 2005; Bole \& Dinely, 1992)

While each of the above factors is significant in this paper, we focused on RCS. It is essential to analyze each factor in terms of detecting targets under different conditions. A further result which follows from the equation is that, in general, the signal strength received varies inversely with the fourth power of the range of the reflecting surface. (This can be shown by replacing $S_{\min }$ with the more general term $S_{r}$ representing received signal strength and then making $S_{\mathrm{r}}$ the subject of the equation.)

It is thus important to appreciate that, for distant targets, a small increase in the range may produce a comparatively large decrease in response. The response from almost all wanted targets follows this law, but some unwanted target responses such as those from the sea and from precipitation did not (Bole \& Dinely, 1992). In the next section, we present the target characteristics.

\section{Target Characteristics}

Energy in the pulse which is intercepted by the target is then available for return towards the antenna and hence to the receiver, which is called a receptive state. The amount of energy which is returned toward the 
antenna, as opposed to that energy which is absorbed and scattered by the target, is dependent upon the following five prime characteristics of the target.

\section{Aspect}

The simplest approach for measuring the target response aims to consider the energy seculars reflection, i.e. the sort of reflection that occurs when light strikes a plain mirror. Aspect is the angle which the radar rays make with the plane of the mirror. Thus, the response will be rated as good when the aspect is $90^{\circ}$; otherwise, it will be rated as poor for all other angles.

\section{Surface Texture}

The extent which the reflection is speculative is dependent on the surface texture of the target, i.e. whether the surface is 'rough' or 'smooth'. Whether a surface is rough or smooth is related to the wavelength of the waves that are striking it. A surface may reflect light poorly due to the fact that the indentations or the facets in the surface have the same order as the wavelength of the light (approximately $0.001 \mathrm{~mm}$ ). This appears smoothly through radar waves whose wavelength (some 3 to $10 \mathrm{~cm}$ ) and specular reflection is shown. It should be noted that objects that appear rough by radar waves, and which scatter a large proportion of the waves, can occasionally improve the response from a target which has an intrinsically poor aspect.

\section{Material}

In general, materials which are good conductors of electricity also return good radar response. This occurs as a result of absorption and reradiation of the waves at the same wavelength as those received, rather than from simple seculars-type reflection.

Some bodies absorb radiation, but when they re-radiate, the wavelength becomes different from the one at which it was received. Despite that, other bodies absorb radiation and re-radiate very little of the energy (this results in the temperature of the body rising, i.e. the received radiation is converted by heat). Some materials are simply transparent to radar energy. GPR behaves to a large extent in this way; steel will return good responses, while wooden boats generally produce poor responses (Lav Varshney, 2002; Marie-Louis et al., 2001).

\section{Shape}

It is frequently suggested that the shape might be considered as a variable aspect of an object. This is true. However, when attempting to assess the degree of response which one might expect from a particular target, it is often convenient to consider the target as a fixed shape that has 
the ability to change its aspect (e.g. a conical buoy rolling in a sea way. This, however, can be seen in Figure 1.
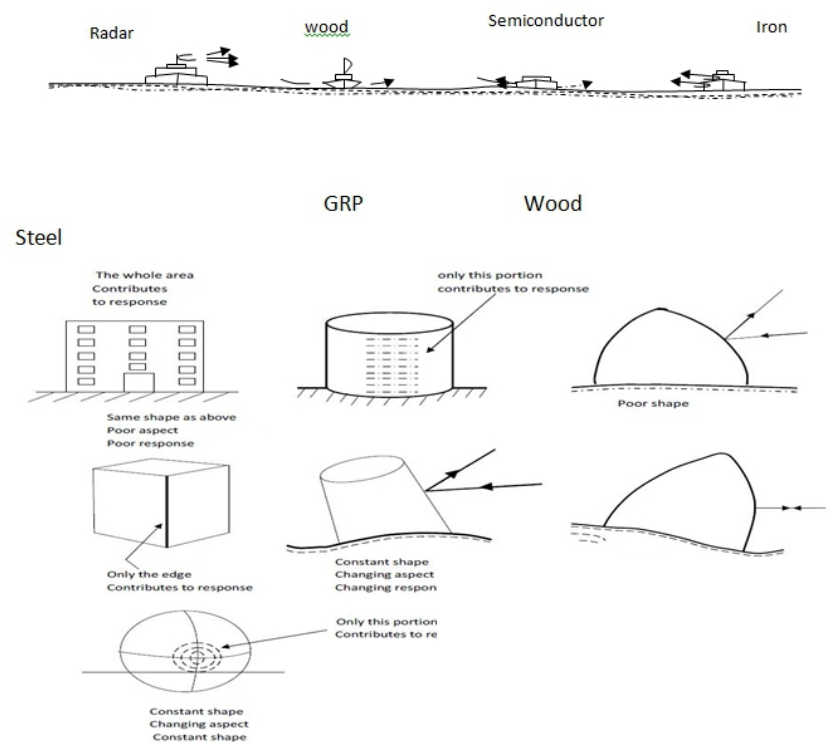

Figure 1. The effect of target on radar

\section{Radar Cross Section (RCS)}

Radar cross section is the measure of a target's ability to reflect radar signals in the direction of the radar receiver, i.e. it is a measure of the ratio of backscatter power per steradian (unit solid angle) in the direction of the radar (from the target) to the power density that is intercepted by the target (George W. Stimson, 2001). However, the RCS of a target can be viewed as a comparison between the strength of the reflected signal from a target to the reflected signal from a perfectly smooth sphere of cross sectional area of $1 \mathrm{~m}$ as shown in Figure 2.

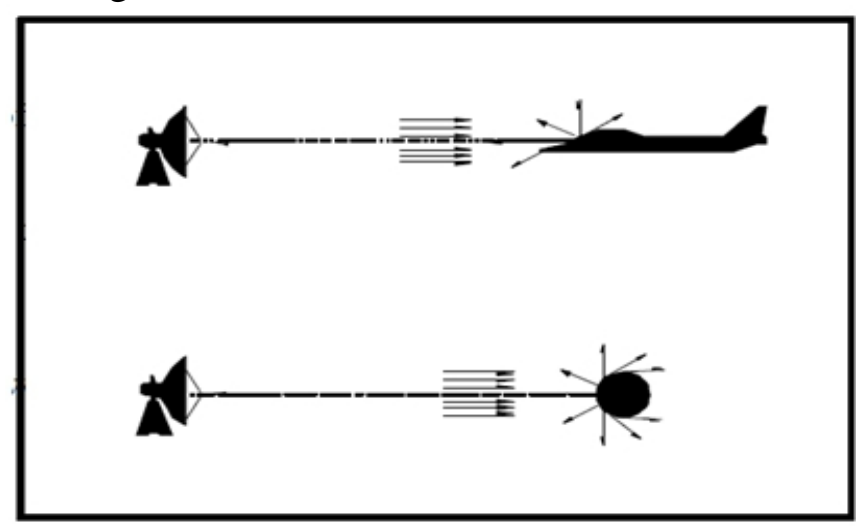

Figure 2. Concept of Radar Cross Section 
The conceptual definition of RCS includes the fact that not all of the radiated energy falls on the target. A target's RCS ( $\boldsymbol{\sigma})$ is most easily visualized as the product of three factors:

\section{$\sigma=$ Project cross section $x$ Reflectivity $x$ Directivity}

$\operatorname{RCS}(\boldsymbol{\sigma})$ is representing power reradiated from the target.

Reflectivity: The percent of intercepted power reradiated (scattered) by the target.

Directivity: The ratio of the power scattered back in the radar's direction to the power that would have been backscattered had their scattering to be uniform in all directions (i.e. isotropically).

Experimentally, radar received the echo signal from a sphere which has a projected area of one square meter (i.e. diameter of about 44 in). Using the spherical shape is of significant importance in field or laboratory measurements since orientation or positioning of the sphere will not affect radar reflection intensity measurements as a flat plate would. When calibrated, other sources (cylinder, flat plate, or corner reflector, etc.) could be used for comparative measurements.

Furthermore, the sphere is essentially the same in all directions. The flat plate has almost no RCS except when aligned directly towards the radar. The corner reflector has RCS almost as high as the flat plate but is over a wider angle, i.e., over $\pm 60 \mathrm{E}$. The return echo signal from a corner reflector is analogous to a flat plate. Thus, this signal is always perpendicular to collocated transmitter and receiver. Targets such as ships and aircraft often have many effective corners. Corners are sometimes used as calibration targets or as decoys, i.e. corner reflectors (Barrie Billingsley, 2002).

An aircraft is a very complex target. It has many reflecting elements and shapes. However, the RCS of real aircraft must be measured. It varies significantly depending on the direction of the illuminating radar. This is shown in Figure 3 below.

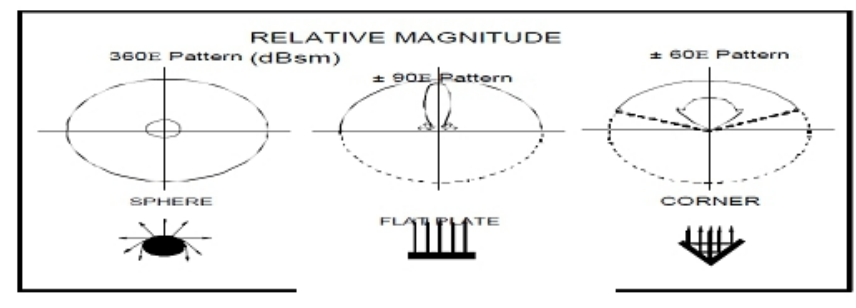

Figure (3) RCS Pattern 


\section{Effect of Target Reflection on Radiation Pattern of Radar Antenna}

In this section, we study the effect of RCS on antenna pattern of the radar receiver for two different directions: (i) first, by taking RCS with different shapes and fixed areas, and (ii) second, by taking RCS with different shapes and areas. The radar antenna, the receiver, and the transmitter used in this operation have specific characteristics. These characteristics are shown below:

\section{1- Parabolic Antenna (Transmitter)}

- $\quad$ Frequency that it uses to transmit (X-band) is 8.5 GHZ.

- $\quad$ The signal wavelength $(\lambda)$ equal to $0.035 \mathrm{~m}$.

- $\quad$ The gain of the antenna $\left(G_{t}\right)$ equal to $28 \mathrm{~dB}$.

- $\quad$ The effective area of the antenna( $\left(\mathrm{A}_{\mathrm{e}}\right)$ equal to $9.75 \times 10^{-5} \mathrm{~m}^{2}$

\section{2- $\quad$ Microstrip Patch Array Antenna (Receiver)}

- $\quad$ Frequency that it uses to receive (X-band) is 8.5 GHZ.

- $\quad$ The gain of the antenna $\left(\mathrm{G}_{\mathrm{r}}\right)$ equal to $15 \mathrm{~dB}$.

- $\quad$ The effective area of the antenna $\left(\mathrm{A}_{\mathrm{e}}\right)$ equal to $9.75 \times 10^{-5} \mathrm{~m}^{2}$

\section{The Effect of Target Shape Equal Area on Antenna Pattern of Radar}

In this section, we consider different forms for RCS that have different shapes and equal areas (approximately $125 \mathrm{~cm}^{2}$ ). These forms are illustrated in Figure 4. At the beginning, we plot pattern for radar antenna in the Cartesian coordinate and polar coordinate in order to specify the shape of the pattern and its maximum at any angle. Figure 5 illustrates the maximum pattern which is $-25.7 \mathrm{dBm}$ at the angle (zero). Furthermore, we selected the radar transmitted antenna with a pencil pattern and narrow beam width to make sure that the maximum power hits the target in order increase the detectability of the radar.

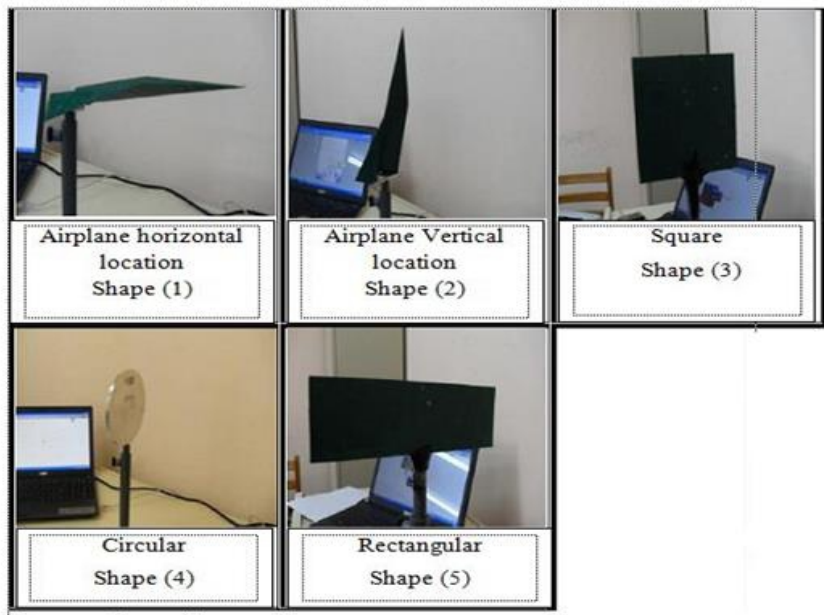

Figure 4. Different form of RCS with equal area 


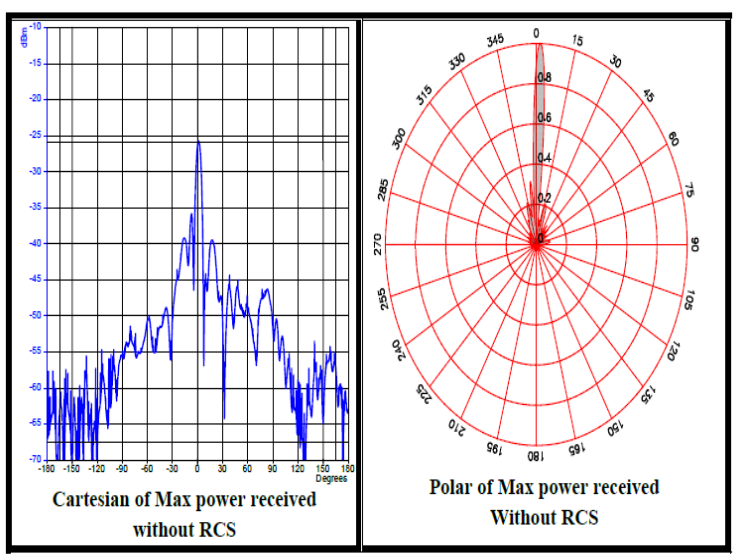

Figure 5. Cartesian and polar coordinates for transmitting antenna of radar

After drawing the pattern for radar antenna, we have to fix the RCS given in Figure 4. This is done one after another at approximately one meter distance in between. Also, the receiver is located close to the transmitter as illustrated in Figure 6. After that, we draw the effect of RCS on the radar antenna using polar and Cartesian forms in order to estimate the dispersion of RCS on pattern for the radar antenna. In other words, the RCS returns the echo signal at the same direction of the receiver antenna.

In this way, we can make the target more detectable for the echo signal in a direction far away from the receiver. However, this makes the detection process harder. Also, it can help in classifying the forms into two categories: (1) forms that make the detection process harder, and (2) forms that make the detection process easier.

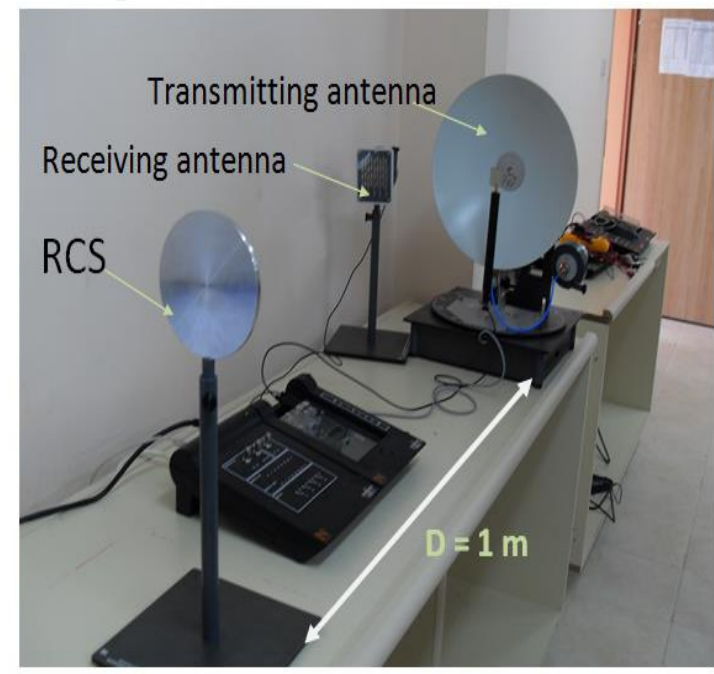

Figure 6. System components 


\section{The First Shape (Airplane)}

However, we selected the first shape as shown in Figure 4, and drew a pattern for the echo signal return from the receiver using the polar and Cartesian forms as illustrated in Figure 7. From this figure, we can conclude that the maximum pattern for the echo signal horizontally is $-37 \mathrm{dBm}$ at angle $340^{\circ}$, and vertically is $-39 \mathrm{dBm}$ at angle $334^{\circ}$.
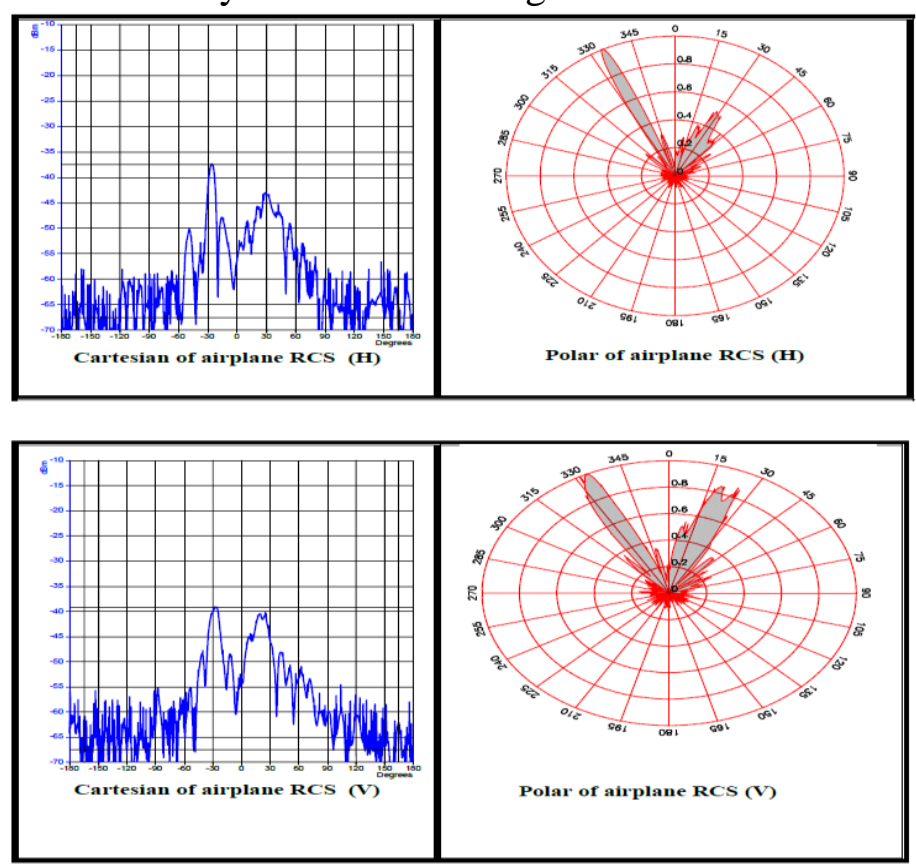

Figure 7. Cartesian and polar coordinate of horizontal and vertical airplane

In this case, the pattern of the echo signal for horizontal location is reflected into two directions with two angles $\left\{\left(340^{\circ}\right),\left(30^{\circ}\right)\right\}$ from the receiver antenna. For the vertical location, it is also reflected into two directions with two angles $\left\{\left(334^{\circ}\right),\left(25^{\circ}\right)\right\}$ from the receiver antenna. This makes the type of RCS to be a powerful dispersion of electromagnetic waves in two directions. Consequently, this makes the target detection process harder.

\section{The Second Shape (Square)}

We selected the second shape as shown in Figure 4. In addition, we drew a pattern for the echo signal return from the receiver using the polar and Cartesian forms as illustrated in Figure 8. From this figure, we can conclude that the maximum pattern for the echo signal is $-42 \mathrm{dBm}$ at the angle $3^{\circ}$. 


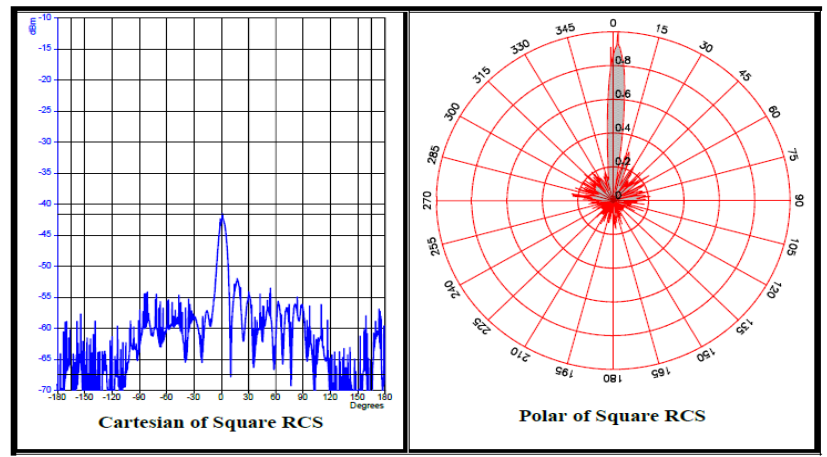

Figure 8. Cartesian and polar for square

In this case, the pattern of the echo signal is slightly away from the receiver antenna at angle $\left(3^{\circ}\right)$. Therefore, this makes this type of RCS to be acceptable as a dispersion of electromagnetic waves.

\section{The Third Shape (Circle)}

We selected the third shape as shown in Figure 4. Also, we drew a pattern for the echo signal return from the receiver using the polar and Cartesian forms as illustrated in Figure 9. Based on this figure, we can conclude that the maximum pattern for the echo signal is $-34 \mathrm{dBm}$ at the angle $\left(3^{\circ}\right)$.

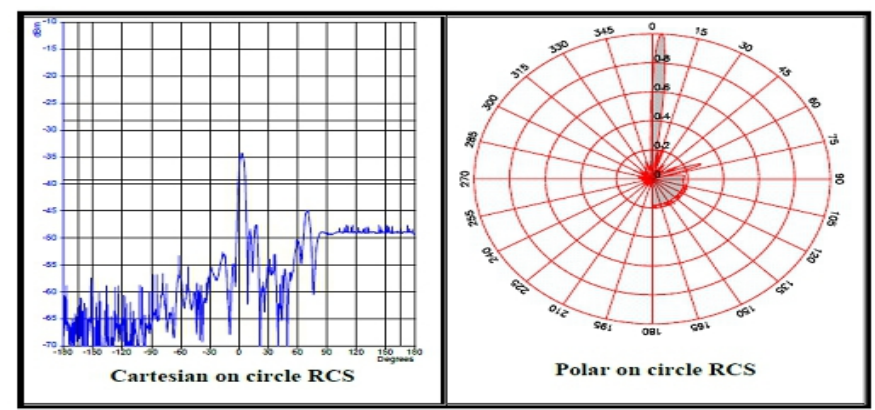

Figure 9. Cartesian and polar for circle

In this case, the pattern of the echo signal is slightly away from the receiver antenna at angle $\left(3^{\circ}\right)$. Therefore, this makes this type of RCS to be acceptable as a dispersion of electromagnetic waves.

\section{The Fourth Shape (Rectangular)}

We selected the fourth shape as shown in Figure 4. Also, we drew a pattern for the echo signal return from the receiver using the polar and Cartesian forms as illustrated in Figure 10. From this figure, we can conclude that the maximum pattern for the echo signal is $-34 \mathrm{dBm}$ at the angle $\left(5^{\circ}\right)$. 


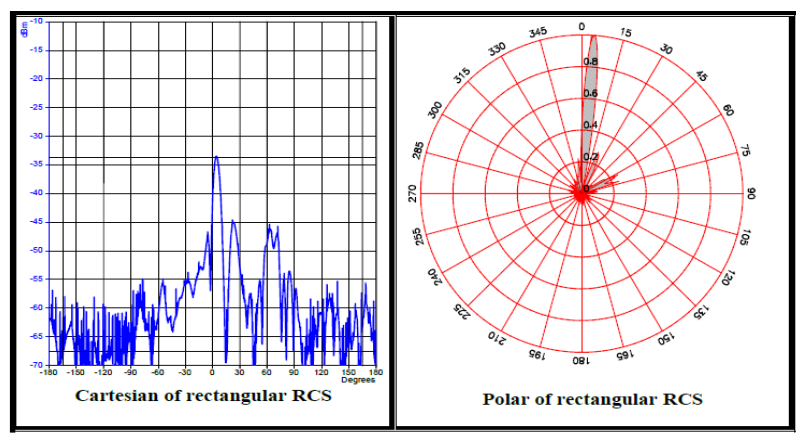

Figure 10. Cartesian and polar for rectangular

In this case, the pattern of the echo signal is slightly away from the receiver antenna at angle $\left(5^{\circ}\right)$. This makes this type of RCS to be acceptable as a dispersion of electromagnetic waves.

\section{The Effect of Different Area Target Shape on Antenna Pattern of Radar}

In this section, we consider different forms for RCS that have different shapes and different areas. These forms are illustrated in Figure 11. At the beginning, we plot pattern for radar antenna in the Cartesian coordinate and polar coordinate in order to specify the shape of the pattern and its maximum at any angle. Figure 5 illustrates the maximum pattern which is $-25.7 \mathrm{dBm}$ at the angle $\left(\mathrm{O}^{\circ}\right)$.

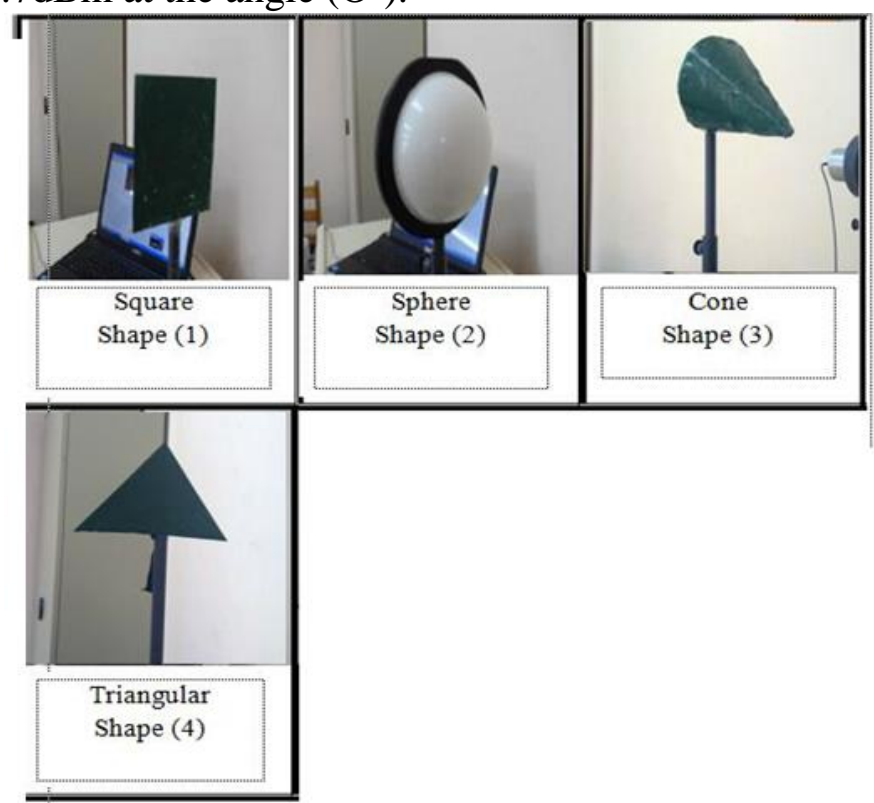

Figure 11. Different form of RCS with different area 
After drawing the pattern for radar antenna, we have to fix the RCS given in Figure 11. It was fixed one after another at approximately one meter distance in between. The receiver is located close to the transmitter as illustrated in Figure 6. After that, we draw the effect of RCS on the radar antenna using Polar and Cartesian forms in order to estimate the dispersion of RCS on pattern for the radar antenna.

Through this way, we make the target more detectable or it deviate the echo signal in a direction far away from the receiver which makes the detection process harder. Furthermore, it can help in classifying the forms into two categories: (1) forms that make the detection process harder and (2) forms that make the detection process easier.

\section{The First Shape (Square)}

We selected the first shape as shown in Figure 11. Also, we drew a pattern for the echo signal return from the receiver using the polar and Cartesian forms as illustrated in Figure 12. From this figure, we can conclude that the maximum pattern for the echo signal is $-38 \mathrm{dBm}$ at the angle $\left(85^{\circ}\right)$.

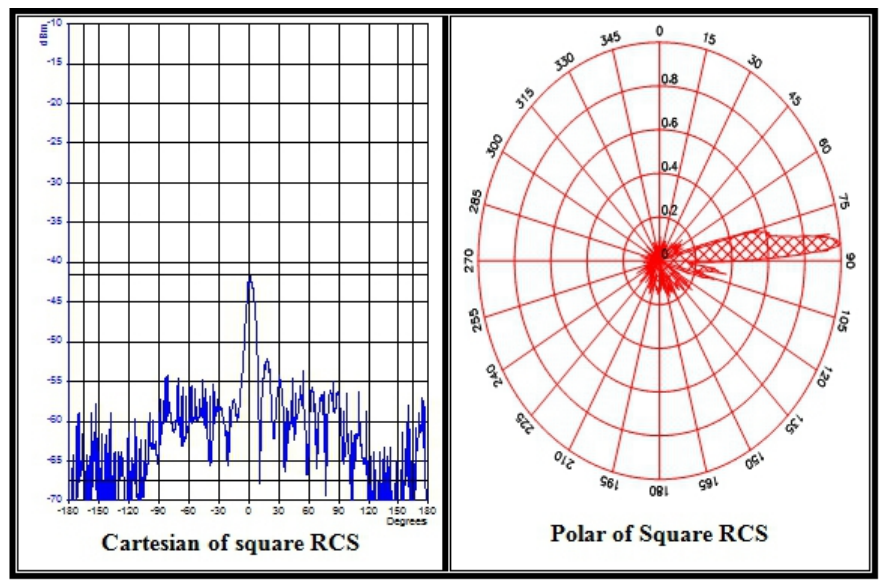

Figure 12. Cartesian and polar for Square

\section{The Second Shape (Sphere)}

We selected the second shape as shown in Figure 11. Also, we drew a pattern for the echo signal return from the receiver using the polar and Cartesian forms as illustrated in Figure 13. From this figure, we can conclude that the maximum pattern for the echo signal is $-38 \mathrm{dBm}$ at the angle $\left(0^{\circ}\right)$. 


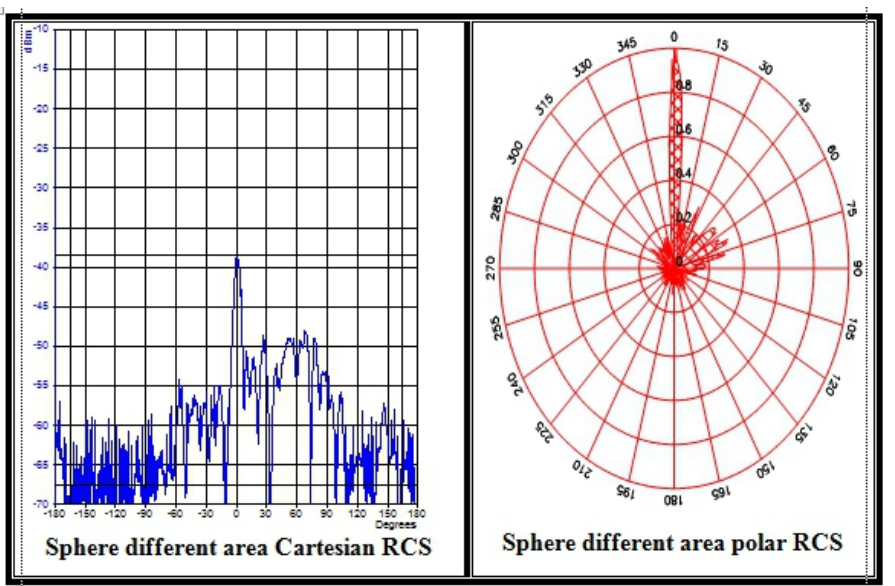

Figure 13. Cartesian and polar for Sphere

We observed that the pattern in the two forms (i.e. Polar and Cartesian) are approximately identical form. This means this type of RCS has a weak effect on the pattern of radar antenna, which does not disparate the electromagnetic waves reflected from this body (i.e. RCS) in the direction of the radar receiver. This will help in making the detection process easier for this type of RCS by the radar.

\section{The Third Shape (Cone)}

We selected the third shape as shown in Figure 11. Also, we drew a pattern for the echo signal return from the receiver using the polar and Cartesian forms as illustrated in Figure 14. From this figure, we can conclude that we have two maximum patterns: the first echo signal is $-38 \mathrm{dBm}$ at the angle $\left(295^{\circ}\right)$ angles, and the second echo signal is $-40 \mathrm{dBm}$ at the angle $\left(340^{\circ}\right)$.

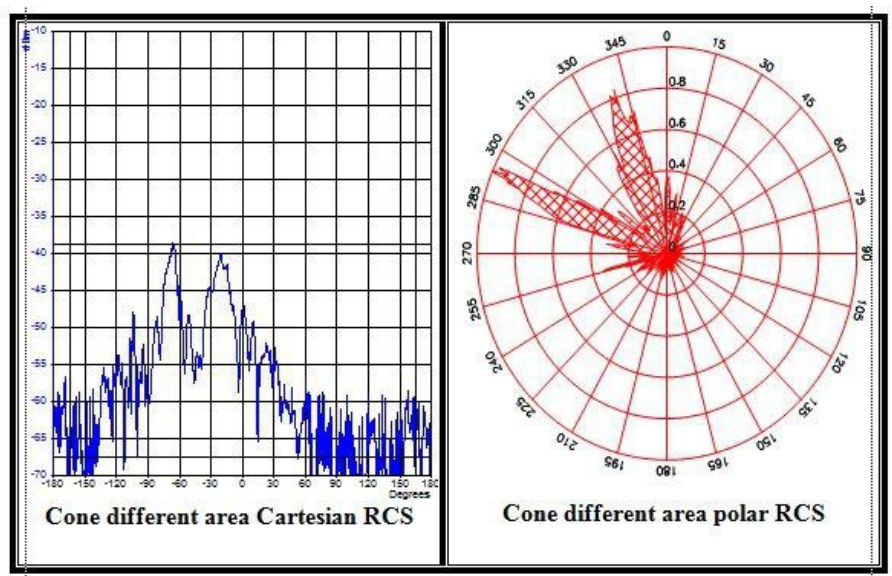

Figure 14. Cartesian and polar for cone 
In this case, the pattern of the echo signal is reflected in two directions with two angles $\left\{\left(295^{\circ}\right),\left(340^{\circ}\right)\right\}$ from the receiver antenna. This makes the type of RCS a powerful dispersion of electromagnetic waves in two directions. Thus, the target detection process becomes harder.

\section{The Fourth Shape (Triangle)}

We selected the fourth shape as shown in Figure 11. Also, we drew a pattern for the echo signal return from the receiver using the polar and Cartesian forms as illustrated in Figure 15. From this figure, we can conclude that we have two maximum patterns: the first echo signal is $-38 \mathrm{dBm}$ at the angle $\left(355^{\circ}\right)$ angles, and the second echo signal is $-42 \mathrm{dBm}$ at the angle $\left(50^{\circ}\right)$.

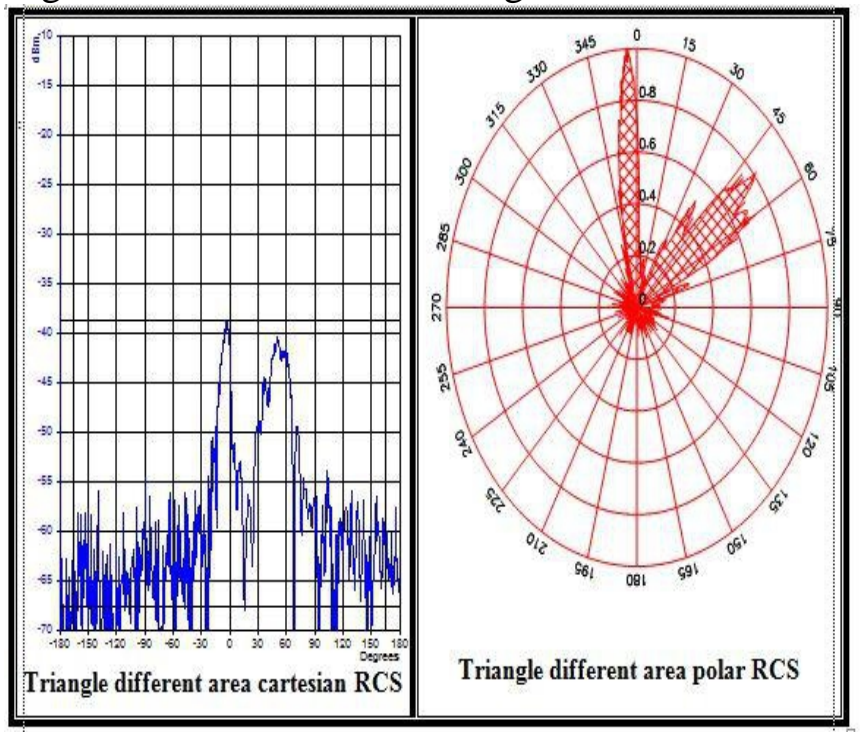

Figure 15. Cartesian and polar for Triangle

In this case, the pattern of the echo signal was reflected in two directions with two angles, $355^{\circ}$ and $50^{\circ}$, from the receiver antenna. This makes this type of RCS a powerful dispersion of electromagnetic waves in two directions. Also, this makes the target detection process harder. In addition, it also makes this type of RCS acceptable as a dispersion of electromagnetic waves.

\section{Conclusion}

In this paper, we tested the effect of RCS on radar detection under two features of RCS using lab radar. However, those features include: (i) shapes with constant area and (ii) shapes with different areas. Thus, the results show that whenever the power of echo signal is high (i.e. RCS is large), the radar detection range becomes high and vice versa.

Based on the results of this study, we concluded on the following: 
1. For features (i) above is rated as good for airplane RCS, and it gives less echo power in the direction of the radar. This means that this shape act as disputer to the electro-magnetic incident waves away from the receiver.

2. For features (ii) above is rated as good for cone and triangular RCS, and they give less echo power in the direction of the radar. This means that those shapes act as disputer to the electro-magnetic incident waves away from the receiver.

This fact can be used in designing the target bodies using different shapes (i.e. reflectors) to dispute the transmitted signal and to weaken it. Therefore, this makes the detection of targets extremely hard.

\section{References:}

1. Barrie Billingsley J. (2002). "Low angle radar land clutter", second edition, United State of America.

2. Bole A.G \& Dinely W. O. (1992). "Radar and ARPA manual", Second edition.

3. Fred E., Nathanson J., Patrick Reilly and Marvin N. cohen (2005). "Radar design principle", Asoke k.ghosh, London.

4. George W. Stimson (2001). Introduction to Airborne radar. Second edition. Printed in United States of America.

5. Lav Varshney (2002). "Radar System Components and System Design", First edition, North Syracuse.

6. Marie -Louis Freysz \& Rodger hickman (2001). "Air and space burne Radar systems", First edition, William Andrew.

7. Merrill I. Skolnik (2002). "Introduction to radar system", third edition, Mc Graw- Hill, printed, America.

8. http://www.radartoutrial.com/radar/radarcrosssectiontarget

9. http://www.wikipedia.org/radar/radarandcrosssection/fundamentals 